\title{
Apreciación del papel educativo de la enfermera en habitantes de una comunidad
}

\author{
Paulín-García, Cecilia*; Gallegos-Torres, Ruth Magdalena
}

\section{RESUMEN}

Introducción: La Educación para la Salud es una de las aspiraciones más fuertes para luchar por la equidad y pretender alcanzar una población saludable con autonomía y capacidad de elección, donde el profesional de Enfermería tiene un papel protagónico. Objetivo: explorar la percepción de los usuarios de un centro de salud sobre el papel de la enfermera en la EpS. Metodología: Estudio cualitativo basado en la Teoría Fundamentada realizado en un centro de salud de una comunidad ubicada en Pie de Gallo, Santa Rosa Jáuregui, perteneciente al Estado de Querétaro. La recolección de datos se hizo mediante entrevista semiestructurada en 2 grupos focales. Se respetaron los principios éticos del Consejo de Organizaciones Internacionales en Ciencias Médicas. Resultados: Se entrevistaron a 20 usuarios (hombres y mujeres). Se obtuvieron 3 categorías de análisis: a. Actitud de la Enfermera hacia los usuarios, b. Cuidados de Enfermería y c. Hallazgos adicionales. Conclusiones: si bien se identifican las funciones primordiales del personal dentro de la clínica, es necesario profundizar en el área educativa dentro de la promoción a la salud.

Palabras clave: Educación en Salud; Enfermeras de Salud Pública; Percepción (DeCS).

'Estudiante de la Maestría en Enfermería, Facultad de Enfermería, Universidad Autónoma de Querétaro. Email: cecepaulin@gmail.com. ORCID: 0000-0002-8295-200X

²Doctora en Ciencias de la Salud, Docente de Tiempo Completo, Maestría en Enfermería, Facultad de Enfermería, Universidad Autónoma de Querétaro. Email: isisrmgx@gmail.com. ORCID: 0000-0001-8034-4089

Recibido: 30/05/2019

Aceptado: 09/12/2019

*Autor para correspondencia

\section{Cómo citar este artículo}

Paulín-García C, Gallegos-Torres RM. Apreciación del papel educativo de la enfermera en habitantes de una comunidad. SANUS. $2019 ;$ (12): 17-33.

[Acceso____ ]; Disponible en: $\frac{}{\text { URL }}$. 


\title{
Apreciação do papel educacional da enfermeira sobre os habi- tantes de uma comunidade
}

\begin{abstract}
ABSTRATO
Introdução: A Educação para a Saúde é um dos anseios mais fortes para lutar pela equidade e pretender alcançar uma população saudável com autonomia e capacidade de eleição, onde o profissional de Enfermagem tem um papel protagônico. Objetivo: Explorar a percepção dos usuários de um posto de saúde sobre o papel da enfermeira na em Educação em Saúde. Metodologia: Estudo qualitativo apoiado na Teoria Fundamentada realizado em um Posto de saúde de uma comunidade localizada em Pie de Gallo, Santa Rosa Jáuregui, no estado de Querétaro, México. A colheita de dados foi feita mediante uma entrevista semi-estruturada em dois grupos focais. Foram respeitados os princípios éticos na pesquisa. Resultados: Foram entrevistados vinte usuários (homens e mulheres). Foram obtidas três categorias de análise: a. Atitude da Enfermeira em relação aos usuários, b. Cuidados de Enfermagem e c. Descobertas adicionais: Há um evidente interesse pela necessidade de contar com mais pessoal de saúde, para uma melhor atenção. Conclusões: Mesmo se foram identificadas as funções primordiais do pessoal dentro do Porto de saúde, é necessário aprofundar na área educativa dentro da promoção da saúde.
\end{abstract}

Palavras chave: Educação na Saúde; Enfermeiras da Saúde Pública; Percepção (DeCS).

\section{INTRODUCCIÓN}

La Educación para la Salud (EpS) se define como un "proceso que informa, motiva y ayuda a la población a adoptar, mantener prácticas y estilos de vida saludables; propugna por los cambios ambientales necesarios para facilitar estos objetivos" (1). Si bien no es un área nueva dado que siempre ha sido parte de la labor enfermera el promocionar la salud en la población, actualmente ha cobrado mayor interés en el ámbito de la promoción de la salud dada la situación mundial en salud, en donde la labor en el primer nivel de atención cobra vital importancia. En este sentido, ha sido propuesta como una de las aspiraciones más fuertes para luchar por la equidad y pretender alcanzar una población saludable con autonomía y capacidad de hacer elecciones adecuadas para la propia salud. Sin embargo, la falta de resultado de dicha aspiración se debe a la concepción de salud centrada en la enfermedad (2).
La ciencia enfermera defiende el estudio y abordaje de la promoción del cuidado y autocuidado individual como parte de su identidad disciplinar; sin embargo, el papel de la enfermería en el autocuidado colectivo no ha sido ampliamente explorado. Este rol está relacionado con la calidad de la relación que se establece entre la enfermera y la comunidad, lo que resulta en la implementación de intervenciones de cuidado y autocuidado con éxito, esto en el contexto en el que hoy en día la ciudadanía exige ser protagonista en la gestión de su salud y en la promoción activa de la misma ${ }^{(3)}$.

La enfermera que se desempeña en servicios clínicos en los diferentes niveles de atención de salud tiene como principal responsabilidad brindar cuidados de enfermería, sin embargo, éstos están estrechamente ligados a la educación para la salud, la cual favorece el desarrollo de capacidades de autocuidado tanto en los pacientes como en las familias para aumentar la autoeficacia en su cuidado (4). 
De acuerdo con el Consejo Internacional de Enfermeras (CIE) y la Organización Mundial de la Salud (OMS), las enfermeras son el eje de los sistemas sanitarios, las cuales desempeñan un papel crucial tanto en la promoción de la salud como en la prevención, tratamiento y cuidados. Las enfermeras muchas veces son el primer $y$, a veces, el único profesional de salud al que la gente puede acceder y la calidad de su evaluación inicial, atención y tratamiento es vital. Son parte de la comunidad local (comparten su cultura, fortalezas y debilidades), pueden dar forma y realizar intervenciones efectivas para cubrir necesidades de los pacientes, familias y comunidades ${ }^{(5)}$. Las funciones de la enfermera dentro del área de educación para la salud son: promoción de la salud, tanto a nivel individual como en lo colectivo, capacitar en medidas preventivas de enfermedades, impulsar la educación para la salud dictando pautas para la adopción de estilos de vida saludables y cambio de hábitos y actitudes para favorecer la salud y calidad de vida, entre otras ${ }^{(6)}$.

La enfermería se identifica como una profesión humanista, centrada en el cuidado individual, colectivo y de entornos, a partir del desarrollo de sus acciones. Sin embargo, estas han sufrido una invisibilización debido a la sobrecarga laboral, la escasa infraestructura institucional, la falta de recursos materiales para la atención y cantidad de personas que se deben atender en un servicio, de acuerdo a Rosa, et al. ${ }^{(7)}$, lo que limita en sí la finalidad misma de un sistema de salud la cual, de acuerdo a la OMS ${ }^{(8)}$ es mejorar la salud de la población, prestando servicios que respondan a las necesidades de la población y sean equitativos, al tiempo que se ofrezca un trato digno a los usuarios.

De acuerdo con lo anterior, la percepción que tienen las personas que acuden a los servicios sanitarios sobre el papel de la enfermera en la EpS depende de la atención recibida y la expectativa que se tiene de la misma. Las enfermeras son el grupo más grande de proveedores de la atención de la salud que están a la vanguardia del cuidado y pasan la mayor parte del tiempo con los pacientes y sus familias, lo que proporciona excelentes oportunidades para otorgar EpS (2).

En este sentido, se identifica que la prioridad es conocer la percepción de los usuarios sobre los sistemas de salud, esto con la finalidad de contribuir en la mejora de la calidad de la atención o de un área en específico dentro del misma. Algunos estudios enfocados a la percepción del usuario del sistema de salud (haciendo mención que dentro del área de atención primaria la información es escasa) que confirman el valor de la atención por parte del área de enfermería, son los siguientes:

En el estudio sobre la "Percepción sobre la atención de enfermería en una unidad de cuidados intensivos", obtuvo como resultado la importancia de la atención de enfermería dentro de esta área para los pacientes, quienes se sintieron seguros y confiados, lo que establece una diferencia entre una experiencia positiva o negativa (6).

En la investigación acerca de la "Percepción del usuario del trato digno por enfermería en un hospital del Estado de México"; se encontró que la satisfacción del usuario de los servicios de enfermería es directamente proporcional a la percepción del trato digno que recibió por este profesional de la salud (9). Asimismo, en la "Percepción de los pacientes acerca de la profesión de enfermería en atención primaria en Tenerife" donde los pacientes reconocen que las enfermeras están a su disposición, ofreciendo un servicio que mejora su salud (10).

Con base en lo planteado, la afinidad que demuestra la enfermera respecto a las demandas y necesidades de la ciudadanía está medida por las apreciaciones que la comunidad y el sistema de salud le confieren, por lo que conocer la opinión del usuario se convierte en una herramienta vital dentro del área de salud, ya que es un indicador importante para determinar la calidad de la atención de acuerdo a la información que ofrecen los usuarios a través de entrevistas o grupos de discusión, por lo que identificar esta apreciación mediante el uso de la investigación cualitativa sobre el papel de enfermería dentro de esta área aporta datos relevantes y complementarios tanto en atención primaria como para servicios hospitalarios, además de ser de suma importancia para la propia práctica enfermera. Debido a esto, surge la necesidad de descubrir el punto de vista de los habitantes de una comunidad sobre el trabajo de enfermería.

En este contexto, se utiliza la Teoría Fundamentada (TF) quien, a través del interaccionismo simbólico realiza un acercamiento con la realidad del objeto de estudio; es una herramienta de análisis que genera, formaliza y hace crecer el conocimiento de la profesión a partir de los hallazgos, con lo que contribuye a la práctica enfermera. Ayuda a identificar la subjetividad y efectos derivados de la interacción entre los actores como punto central de la mirada sociológica, por lo que las preguntas en estudios basados en esta perspectiva van dirigidas al significado que tienen las cosas para los individuos, y la manera en que se afectan sus conductas y la interacción de unos con otros ${ }^{(11)}$.

Así, con base en el referente planteado, es que se propuso como objetivo de esta investigación el determinar la percepción del papel educativo de la enfermera en habitantes de una comunidad del Estado de Querétaro, México.

\section{METODOLOGÍA}

El diseño de la investigación fue de carácter cualitativo basado en la Teoría Fundamentada (TF) utilizando como referente el interaccionismo simbólico. La población de estudio incluía a hombres y mujeres con residencia en una comunidad rural ubicada en el centro-norte de México. Dicho lugar cuenta con 4,089 habitantes y se localiza a 7.8 kilómetros del centro de Santa Rosa Jáuregui. La comunidad cuenta con los servicios básicos de vivienda e infraestructura, además de pavimento en la calle principal. 
El centro de salud cuenta con una recepción, en el cual la enfermera tiene su área de trabajo, dos consultorios médicos, una bodega y un salón independiente del edificio principal donde la población recibe pláticas como parte del apoyo de algunos programas de gobierno. El personal de salud que labora de lunes a viernes de 8:00 am a 3:30 pm, se comprende de una enfermera (quien además se encarga del área administrativa), un médico general y un dentista. El promedio de consultas por día varía (esto de acuerdo a las indicaciones del médico) llegando a ser entre 20 y 30 consultas. Sin embargo, es importante mencionar que, en ocasiones, la enfermera se ausenta para asistir a reuniones o recoger material para el centro y los usuarios son atendidos por el médico o dentista, de acuerdo al tipo de consulta que se requiera.

Para participar en la investigación, los participantes requerían haber referido su asistencia al Centro de Salud en el último año, no importando que supieran leer o escribir, con edades comprendidas entre los 20 y 65 años, con disposición voluntaria para participar en el grupo focal mediante un consentimiento verbal, así como la autorización para utilizar la información con fines académicos y de investigación, siempre respetando su confidencialidad y anonimato mediante un pseudónimo.

El tipo de muestreo fue por bola de nieve, procurando la saturación teórica de los datos. Como estrategia para la recogida de la información se utilizó la entrevista semiestructurada en la modalidad de grupo focal, con el propósito de escuchar y analizar información, teniendo como principal objetivo, entender mejor los sentimientos y pensamientos de la gente con respecto a algún tema, problema o servicio (12). Con la finalidad de delimitar el objeto de interés, se diseñó una serie de preguntas, sin embargo, en el transcurso de la entrevista se daba la libertad para expresar lo que los participantes consideraran importante. Para validar las preguntas, se aplicaron dos pruebas piloto a 5 y 7 personas, respectivamente, con las mismas características correspondientes, pero que no formaron parte de la muestra final. Dicho estudio piloto permitió sondear la calidad de las preguntas planteadas a los participantes, con la finalidad de determinar si estas eran claras y permitían la amplia respuesta a las mismas.

Las entrevistas se realizaron en el domicilio de una de las líderes de la comunidad (Pie de Gallo), debido a lo amplio del lugar y su fácil acceso. El periodo de recogida de la información fue del mes de octubre a noviembre del 2018, con establecimiento de las fechas de reunión por parte de las personas de la comunidad en función de sus tiempos y actividades. Al realizar el estudio se consideraron los aspectos éticos señalados en el Reglamento de la Ley General de Salud en materia de investigación y las Pautas Éticas Internacionales para la Investigación relacionada con la Salud en los Seres Humanos, preparadas por el Consejo de Organizaciones Internacionales en Ciencias Médicas (CIOMS) en colaboración con la OMS ${ }^{(13)}$. La investigación fue sometida a revisión por parte del Comité de Bioética de la Facultad de Enfermería y posterior aprobación y registro del mismo, con número de registro 10145. Todos los participantes del estudio tuvieron claro conocimiento de que su participación era voluntaria, que se podían retirar cuando lo requirieran sin explicar motivo y que la información proporcionada sería manejada en forma confidencial. De cada participante no se requirió ningún dato de identificación que facilitara su ubicación.

Cada grupo focal estuvo integrado por 10 personas. Al inicio de cada grupo focal, a los participantes se les explicaba el objetivo y las características de la investigación, así como se solicitó autorización para poder grabar con audio las sesiones. A partir de ese momento se llevaba a cabo la entrevista; una vez finalizada se procedió a transcribir la información de las entrevistas recogidas a través de grabaciones de audio con ayuda del programa Listen N Write. Para el registro de la información en Word, a cada participante se le planteó un pseudónimo.

El método que se siguió para el análisis de contenido e interpretación fue mediante la comparación continua de los datos (MCC), de acuerdo con Corbin \& Strauss ${ }^{(14)}$, con la finalidad de ir dando forma a la tendencia teórica e ir depurando conceptos y significados a partir de la búsqueda de categorías y subcategorías. Igualmente, de manera simultánea se continuó con la codificación de los datos y sus análisis. Esto mediante la codificación abierta, donde se examinaron, compararon y conceptualizaron los datos, formando un código. Después, mediante la codificación axial, los datos se agruparon de acuerdo a la relación entre categorías y subcategorías y, por último, la codificación selectiva donde resultó la categoría principal (que representa el tema central de la investigación).

\section{RESULTADOS}

Se entrevistaron a 20 personas (15 mujeres y 5 hombres). La Tabla 1 muestra los datos sociodemográficos sobresalientes. Del análisis realizado surgieron 3 categorías, las cuales se describen a continuación.

\section{$1^{a}$ Categoría: Actitud de la Enfermera hacia los usuarios}

De acuerdo con la percepción de los usuarios, es evidente la importancia del buen trato y actitud hacia ellos por parte del personal de enfermería al acudir a consulta, e inclusive se considera como una manera de calificarla, determinando si la enfermera es buena o mala, así como la manera de trabajar, como se muestra en el siguiente fragmento:

Sra. Lety: hacer su trabajo muy bien ¿no? para que nosotros como pues los que nos van a atender ehhh tengamos la capacidad de decidir, "esa es una muy buena enfermera, nos trata bien, hace bien su trabajo y colabora con todos nosotros". 
Tabla 1. Características sociodemográficas de los participantes del grupo focal

\begin{tabular}{|l|c|c|}
\hline \multicolumn{1}{|c|}{ Edad } & Mujeres & Hombres \\
\hline \multicolumn{2}{|c|}{ Rango de Edad } \\
\hline 20-30 años & 5 & 1 \\
\hline $31-40$ años & 5 & 1 \\
\hline $60-65$ años & 5 & 3 \\
\hline \multicolumn{3}{|c|}{ Ocupación } \\
\hline Hogar & 4 & 0 \\
\hline Ventas & 1 & 0 \\
\hline Albañil & 0 & 2 \\
\hline Sin oficio & - & 3 \\
\hline & Nivel Educativo & 5 \\
\hline Primaria & 6 & 0 \\
\hline Secundaria & 8 & 0 \\
\hline Preparatoria & 1 & $n=5$ \\
\hline Total & $n=15$ & \\
\hline
\end{tabular}

Fuente: entrevistas realizadas

Consideran que la enfermera debe de presentarse siempre con una buena actitud, siendo la principal característica el respeto:

Sra. Martha: tener una buena actitud ante la gente, a la persona que va a atender, atenderla pues de guena manera, con respeto, pues todas merecemos respeto hasta para atendernos, aunque asi sea gratis, merecemos respeto, tanto de nosotros para ellos, como ellos para nosotros.

Sr. José: pues más que nada, primeramente, la amabilidad ¿no? Como reciben a la clientela o a las personas, más que nada...

Sin embargo, hay ocasiones en que los usuarios justifican una mala atención bajo el argumento del exceso de trabajo.

Sra. Flor: dice mi hermana que un día fue ella y que agarro y que estaban, pero bien estresadas y que le dijo la enfermera de cosas...eso dice mi hermana.

\section{2da Categoría: Cuidados de Enfermería}

Con respecto al conocimiento sobre los cuidados que proporciona el personal de enfermería, los usuarios denotan una noción general sobre esta actividad que desempeñan dentro de su Centro de Salud:

Sra. Mercedes: el pesarnos, tomar la presión, el azúcar en el dedo, dar las fichas de la gente que va a atender el doctor, a veces ponen alguna inyección o la insulina a las que son diabéticas... pues eso en general.
Sra. Guadalupe: aparte también hacen lo que es las, los estudios del Papanicolaou, ehhh lo del DIU, todo eso de cuidarse y aparte aquí dan pláticas para cómo cuidarse uno, todo eso creo que, eso es lo que hacen las enfermeras.

Referente al tema de prevención (rol fundamental) comentan que en ocasiones se les imparten temas de salud acordes a la temporada o necesidad detectada, así como también hacen mención de temas que les gustaría que también se incluyeran, esto para beneficio de la propia comunidad:

Sra. Guadalupe: ellas nos dicen: "no deben de comer esto y esto..." o como por decir los que están diabéticos o de himpertensión, les quitan lo que es el refresco, la azúcar, la sal, todo eso que les hace daño, entonces desde que ella, ella sabe que uno está enfermo de eso, te da un papelito y te dice: "sabes qué, pues te tienes que más o menos cuidar así para que no te pongas más mal...".

Sra. Lety: ahh, ya sé también que nos debe de enseñar: los primeros auxilios (risas) porque isaben qué? hay veces que los necesitamos mucho, mucho eso para apoyar a nuestros hijos y hasta nuestra familia, o hasta una persona que lo necesite y hay veces que eso no nos lo dan.

Dentro del tema de prevención, también hacen mención del tipo de material educativo que suele utilizar el personal para enseñar o ampliar el conocimiento sobre diversos temas de salud, donde las herramientas más 
utilizadas son los carteles y pláticas dentro o fuera del centro de atención:

Sr. Alberto: si, luego hay cartulinas con información pegada en el centro de salud y a veces mientras uno está esperando pus lo ve, en lo que lo pasan a uno y pues si alguien quiere saber más de lo que ahí hay pues le pregunta a la enfermera o al doctor, bueno es lo que a veces yo hago, las veces que he ido para consulta.

Sra. Flor: en pláticas también, nos reúnen a todos y empieza a hablarnos del tema.

Sra. Fernanda: luego van a las escuelas para darles pláticas a los niños, también.

Sin embargo, en las expresiones de los usuarios se muestra la inconformidad respecto a la atención que reciben grupos beneficiados con programas del gobierno, a los cuales dirigen las pláticas informativas:

Sra. Guadalupe: la de aquí lo hacen con las que tienen "Prospera", más que nada, es a las que juntan y en sus grupos es cuando da la reunión y les dice... así las pláticas, las que tocan, este si es de peso, si es de desnutrición o si es de alimentación o si es de diabetis o ya depende de qué enfermedad toque llevar el...este la plática y sobre de eso nos dice.

Sra. Martha: pero a veces no todas contamos con ese apoyo (Prospera) y esas pláticas, a veces nada más cuando vemos, vamos al centro vemos cartulinas con imágenes, o algo así pues uno las lee ¿verdad?, pero a veces, no todas las personas también saben leer, a veces na'mas vemos.

\section{3era Categoría: Hallazgos adicionales}

El sentir de los usuarios al hablar sobre el Centro de Salud donde acuden para recibir atención hace notorio el interés de contar con más personal de salud, esto no siendo exclusivo para el personal de enfermería.

Sra. Lety: si la comunidad está bastante grande y pues como que, sí necesitamos un poquito más de enfermeras, doctores y pues que nos puedan atender, más que nada.

Sr. Rufles: pus yo veo que haya dos enfermeras, yo he ido a consulta y no está, que porque se fue a Santa Rosa o no sé a dónde y no hay quien nos revise y asi los pasa el doctor, hay veces que si adentro nos revisa el doctor como la hace la enfermera, pero otras veces no... mientras afuera esta solo... na'mas la gente esperando que les hable el doctor para entrar.
Esto deriva en no poder contar con la atención médica necesaria para los habitantes de la comunidad:

Sra. María de Jesús: que pongan otro doctor que nos atienda y enfermera, que venga otro para que atienda a toda la gente, a la comunidad porque aquí es muy grande.

Sra. Teresa: a mi me gustaría que hubiera dos turnos de doctores porque nada más hay uno y pues la que alcanzó la primera cita alcanzo y la segunda ya no y en Santa Rosa si hay de dos veces.

Sra. Camila: es que siempre nos mandan igual, nos regresan igual, yo siempre mejor los llevo a Santa Rosa al centro de salud, porque acá no na'mas dicen "es que ya no hay fichas, es que no porque no viene muy grave, vamos a darle a los que vienen más graves, si vienen de la gripa no les pasa nada", eso es lo que siempre nos dicen a uno.

\section{DISCUSIÓN}

El uso de la TF dentro de la investigación, permite contextualizar y comprender mejor la experiencia subjetiva de las personas ante una situación en particular de la cual no se tiene gran información (15), como es el caso de este estudio, por lo que este abordaje permitió develar la percepción de los usuarios de una comunidad sobre el papel de la enfermera en la EpS al relatar las experiencias, comentarios y opiniones a lo largo de las entrevistas.

Para reforzar la importancia del conocer las apreciaciones de los usuarios dentro del sistema de salud, en la investigación sobre "La percepción de la calidad de la atención de los servicios de salud en México: perspectiva de los usuarios", se indica que, a pesar de que en este estudio los resultados en relación a la atención por parte del personal fueron positivos, la variable relacionada con el buen trato fue baja, por lo que reconocen y aconsejan el profundizar en el conocimiento de la perspectiva poblacional y determinar la necesidad de implementar en los servicios de salud acciones para la mejora continua de la calidad de la atención (16).

La categoría "actitud de la enfermera hacia los usuarios" que surgió de las entrevistas realizadas a los participantes, es el resultado de las vivencias y sentimientos que tienen los mismos entrevistados, en el cual consideran que la mayoría de las veces que acuden a consulta el trato o actitud percibida por parte de la enfermera hacia ellos no se basa en el respeto, cordialidad y amabilidad, factor importante dentro de la imagen de la enfermera dentro de la comunidad. Estos resultados son contrarios a los hallazgos con otros estudios (lo más similar posible, aunque no son cualitativos). Se encontró que García ${ }^{(6)}$ dentro de su estudio denominado "Percepción del usuario del trato 
digno por enfermería en un hospital del Estado de México" encontró que la percepción del usuario acerca del trato de la enfermera fue adecuada, en la cual el 91\% de los usuarios aseguran haber sido tratados con respeto.

Dentro de la investigación cualitativa sobre la "Calidad percibida de la atención en salud en una red pública del municipio de Pasto, Colombia" en uno de los resultados en relación a las características atribuidas hacia el personal de salud, estas fueron buenas, sin embargo, también se hace manifiesto que la carga de trabajo puede incidir en el comportamiento de los profesionales, considerándolos groseros o estresados (17). Esto coincide con los resultados arrojados en nuestro estudio donde los usuarios reconocen que en ocasiones el mal trato hacia ellos, en este caso por parte del personal de enfermería, se debe a la excesiva carga de trabajo que tiene, resultando en malas actitudes o acciones hacia ellos.

Por otra parte, en la investigación sobre la "Satisfacción del paciente en el primer nivel de atención médica" se menciona que la satisfacción con el personal de enfermería resulta alta, aunque se considera importante la toma de medidas para incrementar la calidad de la atención para así lograr un aumento en la satisfacción de los usuarios, así como mejorar aspectos de las relaciones humanas, como lo es la comunicación y el trato al paciente, con un enfoque biopsicosocial ${ }^{(18)}$.

En una investigación sobre la "Percepción del familiar y paciente sobre el trato digno otorgado por el personal de enfermería" se encontró que en general los entrevistados manifiestan ser siempre tratados con respeto, aunque señalan que no se alcanza el indicador estándar para la atención o trato digno por parte del personal de enfermería (19). Esto difiere con la investigación denominada "Percepción del paciente de la atención otorgada por el personal de enfermería" donde se señala que más del 50\% de los participantes manifestaron nunca haber sido tratados con respeto durante su atención (20).

De esta manera podemos observar, de acuerdo a los usuarios al referirse a la atención del personal hacia ellos, que entran en función términos como el trato, amabilidad, aclaración de inquietudes y la disposición de ayudar, como factores importantes para calificar como buena o mala la atención recibida.

Referente al apartado de las Funciones de Enfermería, los participantes conocen las actividades fundamentales propias de la enfermera, tales como "toma de presión, toma de azúcar", aplicación de inyecciones, toma de peso y talla, así como el apoyo al médico cuando es necesario.

Este resultado es similar al encontrado por en la investigación realizada en Costa Rica "Percepción de los usuarios y usuarias sobre el quehacer de enfermería en el primer nivel de atención", en el cual las 218 personas entrevistadas coinciden en que la principal función de Enfermería es la toma de presión arterial, pulso, temperatura y respiración (21).
Dentro de un análisis temático referente a "La imagen social de la enfermería: una profesión a conocer" donde engloban aspectos como, qué espera la sociedad de esta profesión y las tareas o funciones que cree que desempeñan, siendo la más relacionada con la profesión el poner inyecciones, curar heridas o ayudar al médico (22).

En relación al concepto de EpS, donde de acuerdo a los usuarios, se lleva a cabo de una manera superficial y en ocasiones a cierta población, Soto ${ }^{(4)}$, realizó una revisión bibliográfica, titulada "La Educación en salud, un elemento central del cuidado de enfermería" para conocer la educación en salud como parte del rol de enfermería en su quehacer profesional, concluyendo que esta se encuentra en un nivel menos importante, lo que se considera como amenaza ante el riesgo de perder una herramienta básica para el cuidado, sobre todo para el nivel primario, donde la EpS es fundamental para el cambio de conductas en salud.

Por último, en la categoría de "hallazgos adicionales", la falta de personal de salud tanto médico como enfermeras es un problema que desde el punto de vista de la comunidad afecta la atención recibida.

Tener disponibilidad inmediata de los servicios de salud cuando enferman, poder acceder a tratamientos médicos y ser atendidos de urgencia, deben ser los pilares básicos del sistema de salud, sin embargo, de acuerdo a las experiencias de los participantes, estos pilares se tambalean.

Alguna de las limitaciones que se tuvieron durante la recolección de datos fueron las siguientes: a) la intención era contar con participantes de ambos sexos por igual, es decir, la misma cantidad de hombres y mujeres, pero debido al horario asignado para las visitas a la comunidad, los usuarios masculinos se encontraban trabajando, lo que dificultó su participación. b) una vez que se modificó el día y hora para realizar el grupo focal y poder contar con la participación de los hombres, se encontró que estos no eran tan participativos como las mujeres, por lo que en el segundo grupo focal se convocaron únicamente a mujeres.

\section{CONCLUSIONES}

Con base al objetivo planteado en el presente estudio se concluye que, dentro de la comunidad, se identifica el quehacer básico de la enfermera, así como el trabajo dentro del área preventiva. Al respecto se reconoce que para cumplir con el propósito y función primordial de enfermería en el área comunitaria es necesario que ésta profundice en la implementación de la promoción a la salud explotando al máximo las herramientas de la EpS, para realmente impactar en la comunidad y lograr que la población se apodere y tome la decisión de modificar su estilo de vida de acuerdo con los recursos a su alcance. En este sentido, los resultados del estudio muestran que, en efecto, la manera en que es apreciada la enfermera impacta en sus relaciones con la comunidad, sin embargo, no se aprecia un significado de alto valor dada la relación superficial que se tiene con la 
misma.

Valdría la pena realizar estudios que permitan conocer el otro lado de la moneda, es decir, la percepción del personal de enfermería sobre su función educadora de la población, su rol dentro de la comunidad y dentro de un equipo multidisciplinario.

\section{CONFLICTO DE INTERESES}

No existe conflicto de intereses entre los autores

\section{FINANCIAMIENTO}

No existe financiamiento para su realización.

\section{REFERENCIAS BIBLIOGRÁFICAS}

1. Pérez MK, Serrano PM, Hernández PK, Fernández BH. Educación para la salud y acciones de enfermería: una articulación en el control del riesgo pre-concepcional. Revista Cubana de Enfermería [revista en internet]. 2016 [citado 2019 Feb 26]; 32 (2). Disponible en: http://www.revenfermeria.sld. cu/index.php/enf/article/view/903.

2. Ocampo RD, Arango RM. La educación para la salud: "concepto abstracto, práctica intangible" [Educaction for health: "Abstract concept, intangible practice"]. Rev Univ. Salud. 2016; 18 (1): 24-33.

3. Colomer-Pérez N, Gea-Caballero V, Chover-Sierra E, Paredes-Carbonell J. Salutogénesis y autocuidado comunitario: también responsabilidad enfermera. Rev Esp Salud Pública. 2018; 92: 1-4.

4. Soto P, Masalan P, Barrios S. La Educación en salud, un elemento central del cuidado de Enfermería [The Health Education a Central Elemet of Nursing Care]. Rev. Med. Clin. Condes. 2018; 29 (3): 288-300.

5. Organización Mundial de la Salud. [Internet]. [Update 27 febrero 2018; cited 2019 febrero]. Available: https://www. who.int/hrh/nursing_midwifery/es/

6. Mendoza RS, Torres BM, Rincón LJ \& Urbina AB. Percepción sobre la atención de enfermería en una unidad de cuidados intensivos. Rev. Enferm. Instituto Mexicano del Seguro Social. 2015; 23 (3): 149-156.

7. Rosa-Eduardo R, Zamora-Monge G. Cuidados invisibles: ¿son suficientemente reconocidos? Index Enferm [Internet]. 2012 [citado 2019 Sep 18]; 21 (4): 219-223. Disponible en: http://scielo.isciii.es/scielo.php?script=sci_arttex\&pid =\$113212962012000300009\&lng=es. http://dx.doi.org/10.4321/
S1132-1296201000300009.

8. Organización Mundial de la Salud. Acerca de los sistemas de salud. Ginebra: OMS; 2005. [cited 2019 septiembre 18]. Available from: http://www.who.int/healthsystems/about/es/.

9. García GC \& Cortés El. Percepción del usuario del trato digno por enfermería en un hospital del Estado de México. Rev. CONAMED. 2012; 17 (1): 18-23.

10. Ramos SS, Ruymán BP, Sánchez NF, Fernández GD. Percepción de los pacientes acerca de la profesión enfermera en atención primaria. 2015. [citado 2019 Feb 26]; 9 (3). Disponible en: http://scielo.isciii.es/scielo.php?script=sci_ arttext\&pid=S1988-348X2015000300018\&Ing=es. http:// dx.doi.org/10.4321/S1988-348X2015000300018.

11. Acevedo-Hernández BA, Moriel-Corral BL, GonzálezCarrillo E, Berumen-Burciaga LV, Pizarro N. Proceso educativo de enfermería para promover el autocuidado de la mujer durante el puerperio. [Nursing educational process to promote self-care of women during the postpartum period]. Rev Enferm Inst Mex Seguro Soc. 2016; 24(3): 197-204.

12. Onwuegbuzie AJ, Dickinson WB, Leech NL \& Zoran AG. Un marco cualitativo para la recolección y análisis de datos en la investigación basada en grupos focales. Paradigmas. 2011; 3: 127-157.

13. Organización Panamericana de la Salud/Consejo de Organizaciones Internacionales de las Ciencias Médicas. Pautas Éticas Internacionales para la investigación relacionada con la Salud con seres humanos. $4^{a}$ ed Ginebra: Consejo de Organizaciones Internacionales de las Ciencias Médicas (CIOMS). 2016.

14. Lenise-do-Prado M, De Souza M, Monticelli M \& Gómez P. Investigación cualitativa en enfermería, Metodología y didáctica. Washington DC. 2013; 24-37.

15. Vivar Cristina G, Arantzamendi M, López-Dicastillo O, Gordo-Luis C. La Teoría Fundamentada como Metodología de Investigación Cualitativa en Enfermería. Index Enferm [Internet]. 2010. [citado 24 marzo 2019]; 19 (4): 283-288. Disponible en: http://scielo.isciii.es/scielo.php?script=sci_ arttext\&pid=S1132-12962010000300011\&lng=es

16. Ramírez-Sánchez T, Najera-Aguilar P, Nigenda-López G. Percepción de la calidad de la atención de los servicios de salud en México: perspectiva de los usuarios. Salud Pública de México. 1998; 40 (1): 3-12.

17. Yépez-Chamorro MC, Ricaurte-Cepeda M, JuradoFajardo DM. Calidad percibida de la atención en salud en una red pública del municipio de Pasto, Colombia. [Perceived quality of health care in a public network in the municipality 
of Pato, Colombia]. Univ. Salud. 2018; 20 (2): 97-110. DOI: http://dx.doi.org/10.22267/rus.182002.114

18. Vázquez-Cruz E, Sotomayor-Tapia J, González-López A, Montiel-Jarquín A. Gutiérrez-Gabriel I, Romero-Figueroa M, Loría-Castellanos J, Campos-Navarroy L. Satisfacción del paciente en el primer nivel de atención médica. [Patient satisfaction in primary medica care in Mexico]. Rev. Salud Pública. 2018; 20 (2): 254-257.\}

19. Ramírez-Muñoz $\vee$, gallegos-Carballo L, Bolado-García PB, Gamboa-López G, Medina-Escobedo CE. Percepción del familiar y el paciente sobre el trato digno otorgado por el personal de enfermería. [Relative's and patient's perception about fair treatment provided by nurses]. Rev. Enferm Inst Mex Seguro Soc. 2016; 24 (2): 109-114.

20.Puebla-Viera DC, Ramírez-Gutiérrez A, Ramos-Pichardo P, Moreno-Gómez MT. Percepción del paciente de la atención otorgada por el personal de enfermería. Rev. Enferm Inst Mex Seguro Soc. 2009; 17 (2): 97-102.

21. Matamoros-Quesada K, Obando-Godínez N, RiveraVolio S, Romero-Guillen D, Torres-Acuña C, Guzmán-Aguilar A. Percepción de los usuarios y usuarias sobre el quehacer de Enfermería en el Primer Nivel de Atención. Rev. Enfermería Actual en Costa Rica. 2007: 12.

22. Errasti-Ibarrondo $B$, Arantzamendi-Solabarrieta $M$, Canga-Armayor N. La imagen social de la enfermería: una profesión a conocer. [The public image of nursing: a profession to learn about]. An. Sist. Sanit. Navar. 2012; 35 (2): 269-283. 